\title{
La emergencia de la movilidad ciclista en las ciudades
}

\section{The emergence of cycling mobility in the cities}

\author{
Ricardo Marqués ${ }^{1}$ y Manuel Calvo Salazar ${ }^{2}$
}

Hábitat y Sociedad (ISSN 2173-125X), n. ${ }^{\circ}$ 13, noviembre de 2020, pp. 5-7. http://dx.doi.org/10.12795/HabitatySociedad.2020.i13.01

1 Catedrático en la Facultad de Física de la Universidad de Sevilla. Responsable del Servicio Integral de la Bicicleta de la Universidad de Sevilla (SIBUS) y presidente de la asamblea ciclista A Contramano. E-mail: marques@us.es. ORCID: 00000003-0528-384X

2 Socioecólogo, especialista en ecología urbana y movilidad sostenible. Como consultor, ha participado desde sus inicios en las políticas de fomento de la bicicleta como medio de transporte aplicadas en Sevilla. Ha sido el coordinador de la redacción del "Programa de la Bicicleta de Sevilla 2020”. ORCID: 0000-0003-2161-5146

3 Por desgracia, en el momento en que se escribieron la mayoría de los artículos de este número especial la crisis de la COVID-19 aún no había estallado o se encontraba demasiado reciente como para incluirla en los análisis. Analizar el impacto de dicha crisis en la movilidad urbana en general y en el ciclismo urbano en particular es ciertamente una tarea pendiente de gran interés.
$\mathrm{H}$ ace ahora aproximadamente 200 años que el barón Karl Drais presentó en la ciudad alemana de Mannheim su laufmaschine o máquina para correr, conocida universalmente como "draisiana". Dos siglos después la draisiana, convertida en la bicicleta moderna, es el modo de transporte más utilizado en el Planeta. Pese a que el ascenso imparable del automóvil desde la década de los sesenta parece haberla relegado a un papel marginal en las políticas de transporte, la bicicleta sigue siendo el vehículo más vendido en el mundo y el vehículo de transporte individual más utilizado por la mayoría de la población mundial.

En las últimas décadas, especialmente a partir de la crisis del petróleo de los setenta, la bicicleta está experimentando, además, un auge inesperado en las ciudades de los países más ricos, como respuesta a las señales que se van acumulando acerca de la crisis medioambiental, de la que la motorización de la movilidad urbana es una de las principales responsables. Adicionalmente, la bicicleta se ha revelado como el medio de transporte social y económicamente más eficaz, lo que contribuye a mejorar sustancialmente los estándares de habitabilidad de las ciudades. En el último año, la crisis sanitaria de la COVID-19 ha supuesto un nuevo impulso a la movilidad ciclista en las ciudades de todo el mundo. ${ }^{3} \mathrm{El}$ presente número de la revista Hábitat y Sociedad trata de contribuir al análisis de esta EMERGENCIA DE LA MOVILIDAD CICLISTA EN LAS CIUDADES, desde diferentes puntos de vista que comparten una inquietud común: Cómo hacer de las ciudades espacios más habitables.

El artículo "Entre infraestructuras y culturas. Discursos y prácticas en torno a la movilidad urbana en Andalucía" de Macarena Hernández-Ramírez y Mario Jordi Sánchez analiza desde una perspectiva multidisciplinar "los diferentes usos y prácticas relativas a la movilidad sostenible en general, y al ciclismo utilitario en particular, en los espacios urbanos de Andalucía, de cara a poder elaborar recomendaciones para fomento del uso de la bicicleta en Andalucía”. Nos ha parecido que constituye una excelente introducción al número ya que ofrece una muy buena panorámica de la situación y de la percepción social de la movilidad ciclista en Andalucía, así como una reflexión general, que excede el ámbito andaluz, y por ello nos parece especialmente valiosa, acerca de los factores sociales que condicionan el éxito de las políticas de promoción de la bicicleta, generalmente centradas, hasta la fecha, en la creación de infraestructuras.

Uno de los aspectos más importantes a la hora de desarrollar planes de fomento de la bicicleta es el análisis de su impacto macro-económi- 
co. El artículo "The economic value of cycling. A methodological assessment for Starter Cities" de João Pedro Ferreira, Frederico Moura Sá, Catarina Isidoro y José Carlos Baptista Da Mota nos propone una metodología para dicho análisis centrada en el caso de Portugal, pero aplicable a muchas otras ciudades del mundo, donde la bicicleta ocupa todavía una posición marginal en la planificación de la movilidad urbana. Factores como el impacto del uso de la bicicleta en la salud pública o en la preservación del medio ambiente deben ser incorporados al análisis económico y el artículo mencionado desarrolla una metodología para ello. El siguiente artículo, "Salud urbana, bicicletas y árboles", de Enrique Figueroa-Luque, Teresa Figueroa-Luque, Elena Mateos Martínez, Teresa Luque Palomo y Enrique Figueroa Clemente, se enmarca precisamente en el análisis de las características que debe presentar una infraestructura ciclista saludable, mientras que el artículo de Francisco Bastida titulado "La Bicicleta y el Estado social de Derecho" analiza el complejo y difícil encaje de la bicicleta como medio de transporte en una legislación de tráfico pensada para el automóvil y la movilidad motorizada, mediante el análisis crítico de su evolución en los últimos años en España.

Se sabe que allí donde la bicicleta ocupa un lugar relevante en las pautas de movilidad, también se produce una mayor presencia de mujeres entre los usuarios de la bicicleta. El artículo de Anna Obach Lapieza y María Ramos Sanz, "Elementos clave para la introducción de la perspectiva de género en las infraestructuras ciclistas", nos recuerda este hecho y nos ofrece un análisis de los factores que posibilitan esa mayor presencia de las mujeres en el ciclismo urbano. Por su parte, el artículo de Edorta Bergua, "La bicicleta en el país de la (auto)movilidad eléctrica", nos recuerda el papel relevante que juega y puede jugar la bicicleta eléctrica de pedaleo asistido en el camino hacia una movilidad urbana más sostenible, un papel que parece haber sido olvidado por las políticas dominantes de fomento de la electrificación de la movilidad, volcadas casi en exclusiva en el fomento del automóvil eléctrico. Finalmente, el artículo "La imagen de la bicicleta en la comunicación publicitaria: movilidad sostenible y cambio climático”, de Gerardo Pedrós-Pérez, Pilar Martínez Jiménez y Pilar Aparicio Martínez, analiza el papel de la bicicleta como elemento publicitario, los mensajes subliminales que suelen acompañar a esta utilización, así como las buenas y malas prácticas que la acompañan.

Cierran el apartado de artículos monográficos de este número especial tres artículos dedicados al análisis de casos concretos, pero de gran relevancia y de los que pueden derivarse importantes enseñanzas prácticas aplicables a otras situaciones similares. El artículo de Pedro Malpica, "La influencia del campo social pro-bicicleta en el proceso de promoción del ciclismo urbano en Sevilla", analiza el complejo proceso de alianzas que posibilitaron el éxito de una iniciativa ciudadana de fomento de la bicicleta. El artículo de Lake Sagaris, Maya Flores y Daniel Lanfranco, "De Rutas Seguras a Rutas Bakanes ${ }^{4}$ en Chile: Co-creación con enfoque de género, educación cívica, y derechos", propone una metodología participativa para ir más allá del concepto de ruta segura y englobarlo en un concepto más amplio, con implicaciones de género y de empoderamiento cívico. Finalmente, el artículo "Contribución al análisis del impacto de la creación de una red de vías ciclistas en la economía local y en la imagen turística de las ciudades. El caso de Sevilla”, de Ricardo Marqués, Manuel López-Peña y Vicente HernándezHerrador, desarrolla y aplica una metodología para el análisis micro-

4 El término 'bakán' (o ‘bacán') se utiliza en Chile y otros países de Latinoamérica para designar algo bueno o divertido. En España podría traducirse como 'guay' o 'guapa'. 
económico de las políticas de fomento de la bicicleta en las economías locales, un aspecto importante a la hora de generar consenso social y forjar alianzas en torno a esas políticas.

El número especial se completa con cuatro artículos en la sección de Lecturas, Eventos y Debates (LED) dedicados al monográfico. El primero de ellos es un estudio de caso que ejemplifica la evolución del tratamiento recibido por la bicicleta en los planes de ordenación urbana en un municipio de Latinoamérica de tamaño medio. Los tres restantes se refieren más directamente al apartado bibliográfico, siendo el primero de ellos la presentación de un centro de documentación en torno a la bicicleta pionero en España y los dos restantes la presentación de libros y colecciones bibliográficas de especial interés para el tema de este número especial.

Se trata en este número, en definitiva, de contribuir a la creación de un marco completo para comprender el papel que la bicicleta ya está jugando en el desarrollo urbano sostenible y la potencialidad que, en el futuro, puede seguir teniendo. Así, la bicicleta propone, más allá de su papel concreto como instrumento de transporte, un modo de mirar y vivir la ciudad. Consideramos que este modo de mirar y vivir estará, está ya, en la base de la conformación de un consenso social proclive a la transformación de nuestro espacio urbano en un espacio más habitable y sostenible.

Marqués, R. y Calvo Salazar, M. (2020). La emergencia de la movilidad ciclista en las ciudades. Hábitat y Sociedad, 13, 5-7.

$<$ http://dx.doi.org/11.12795/HabitatySociedad.2020.i13.01> 


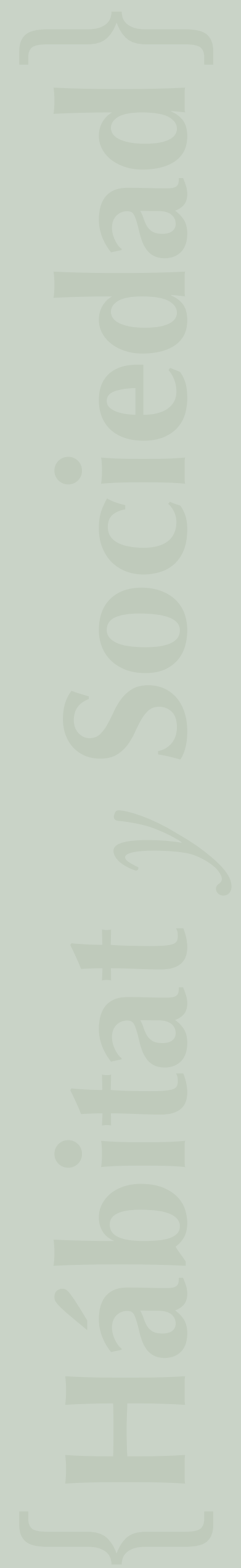

